\section{Hsun-Liang Chan Sharon L. Brooks Jia-Hui Fu Chu-Yuan Yeh Ivan Rudek Hom-Lay Wang}

\section{Authors' affiliations:}

Hsun-Liang Chan, Jia-Hui Fu, School of Dentistry, University of Michigan, Ann Arbor, MI, USA

Sharon L. Brooks, Department of Periodontics and Oral Medicine, School of Dentistry, University of Michigan, Ann Arbor, MI, USA

Chu-Yuan Yeh, School of Dentistry, University of Michigan, Ann Arbor, MI, USA

Ivan Rudek, School of Dentistry, University of Michigan, Ann Arbor, MI, USA

Hom-Lay Wang, Department of Periodontics \& Oral Medicine, School of Dentistry, University of Michigan Ann Arbor, MI, USA. Eng. A.B. Research Chair for Growth Factors and Bone Regeneration, King Saud University, Riyadh, Saudi Arabia

\section{Corresponding author:}

Hom-Lay Wang

IO I I North University Avenue

Ann Arbor, MI 48109-I078, USA

Tel.: + I $734763-3383$

Fax: + I 7349360374

e-mail: homlay@umich.edu

\title{
Cross-sectional analysis of the mandibular lingual concavity using cone beam computed tomography
}

Key words: anatomy, complication, cone beam computed tomography, dental implant, edentulous, mandible

\section{Abstract}

Objectives: To study the prevalence and the degree of lingual concavity in the edentulous first molar region from cone beam computed tomography (CBCT) scans of the mandibles.

Material and methods: Qualified cross-sectional images in mandibular first molar edentulous region taken from CBCT were selected. The mandible morphology $2 \mathrm{~mm}$ above the inferior alveolar canal (IAC) was classified into the convex (C), parallel $(P)$ and undercut $(U)$ type, based on the presence of lingual concavity and the shape of alveolar ridge. The prevalence of each group was determined. Subsequently, the lingual concavity characters, including the depth, the angulation and the vertical location were determined by the measurements of selected anatomic landmarks.

Results: One hundred and three subjects (mean age 51 with a range of 23.7-70.4 years) were studied. The $U$ type was the most prevalent, accounting for $66 \%$ of the study population. The mean undercut depth and angulation at the level $2 \mathrm{~mm}$ above IAC were on average $2.4 \mathrm{~mm}$ and $57.7^{\circ}$. The mean vertical distances from the most prominent point $(P)$ of the lingual concavity to the cemento-enamel junction of second premolar and the inferior border of the mandible were 11.7 and $14.9 \mathrm{~mm}$, respectively.

Conclusions: The anatomic location and the degree of the lingual concavity presented in this article add more information in implant treatment planning in the mandibular first molar edentulous region.

Implant therapy has become an integral part of daily dental practice because of its high success rate. With proper diagnosis and treatment planning, most implant surgeries can proceed uneventfully and fulfill functional and esthetic demands after osseointegration. However, surgical accidents and complications do occur (Greenstein et al. 2008a, 2008b). They can happen during surgery, at the healing period or even after function. They can cause either mild or severe problems, depending on the degree of the damage. Unavoidable complications may be triggered by placing implants outside the osseous boundary (Berberi et al. I993; Givol et al. 2000; Kalpidis \& Setayesh 2004). They may cause damage of vital anatomical structures, resulting in loss of function (Berberi et al. I993) or life threatening events (Givol et al. 2000; Kalpidis \& Setayesh 2004). They may also result in inflammation, infection and ultimate loss of implants at later time (Annibali et al. 2009).

In the posterior mandibular region, a lingual undercut is a common finding and can be difficult to manage. It is not unusual for surgeons to struggle when placing implants in this area, especially when a lingual plate perforation is suspected. A lot of time it is necessary to check the angulations and positioning of the drills or implant fixtures via radiographs and clinical detection of a possible perforation in the osteotomy site. This tedious process increases the length of the surgery and adds stress to both the patient and the clinician thus compromising the success of the procedure.

Some surgeons advocate the use of conventional or computed tomography (CT) for pre-operative implant planning because of the advantages that cross-sectional views bring, for example, clearer visualization of the anatomy of the surgical site (Chen et al. 2008). Actually, the American Academy of Oral and Maxillofacial Radiology (AAOMR) (Tyndall \& Brooks 2000) recommended the inclusion of cross-sectional imaging to evaluate any potential implant site. Nevertheless, the paper also admitted that there is no evidence to support this statement. In fact, indiscriminant use of dental imaging, especially conventional and CT can be potentially harmful to patients, when the cost and radiation doses are considered. Recently, the issue has been raised 
about radiation exposure and potential risk associated with the increasing use of medical CT (Brenner \& Hall 2007). If certain types of bone morphology can be identified during clinical examination, the use of dental imaging can be more selective and efficient. For example, in cases with high risk of lingual plate perforation (e.g. deep lingual undercut), the prescription of cross-sectional imagines could be justified.

Knowledge of anatomical structures and their relations to the site of interest, together with accurate treatment planning of the case, are probably the best way to avoid surgical complications (Greenstein et al. 2008a, 2008b). Lekholm \& Zarb (I985) described five stages of jaw resorption, ranging from minimal to extreme and incorporated this system in implant planning. Their primary focus was volumetric changes of the alveolar ridge after edentulism. Recently, the cross-sectional morphology in the interforaminal area was described in more detail and the presence of lingual concavity and severe slope of lingual cortex were identified as potential risks during surgeries (Tepper et al. 200I; Quirynen et al. 2003). In the posterior mandibular region, detailed information about the lingual concavity seemed scarce. The aim of this article was to study the prevalence and the degree of lingual concavity in the edentulous first molar region using cross-sectional images from cone beam computed tomography (CBCT) scans of the mandibles.

\section{Materials and methods}

This study was approved by the institutional review boards (IRB) of the University of Michigan (09-PAFo4299).

\section{Image acquisition and patient confidentiality}

All images were acquired from a CBCT machine (i-CAT Cone-Beam Computed Tomography machine, Imaging Sciences International, Hatfield, PA, USA) in the Department of Periodontics and Oral Medicine, University of Michigan, School of Dentistry by board-certified Oral and Maxillofacial radiologists. Imaging parameters were set at I $20 \mathrm{kVp}, \mathrm{I} 8.66 \mathrm{mAs}$, scan time $20 \mathrm{~s}$, resolution $0.4 \mathrm{~mm}$ and the field of view (FOV) varied, depending on whether a single arch or both arches were scanned.

CBCT images of each individual were labeled without disclosure of his/her names and personal information. They were transferred to a secured file in a personal desktop computer equipped with an implant planning software program (InvivoDent, Anatomage, San Jose, CA, USA). Data were saved in the Digital Imaging and Commu- nications in Medicine (DICOM) format. A list with the codes and corresponding names was created and saved in an encrypted file so that the patients' confidentiality is protected and yet retrievable if needed.

\section{Inclusion criteria}

All the images used in the study were from the complete patient database of the clinic (20052009) and were not made specifically for this paper. The investigated site was the mandibular edentulous first molar area. With a $4 \times$ ro $\mathrm{mm}$ regular-sized implant as the guide, a calibrated examiner (C. Y.) used the following criteria to select the samples. First, the CBCT images of the mandible had to be available. Second, at least one mandibular first molar had to be absent while the adjacent second premolar was present. Third, the experimental site had to have sufficient vertical bone height ( $\geq \mathrm{I} 2 \mathrm{~mm}$ from the alveolar crest to the superior border of the inferior alveolar nerve canal [IAN]) to possibly place a ro mm implant (Sammartino et al. 2008). Fourth, the experimental site had to have adequate horizontal bone width ( $\geq 3.5 \mathrm{~mm}$ ) (Chiapasco et al. I999).

\section{Assessment of the cross-sectional morphology}

All morphologic assessment and measurements were conducted by one examiner (C. Y.). The qualified CBCT images were opened using an implant planning software program (InvivoDent). The region of interest (ROI) included the mandibular occlusal plane to inferior border. If the second molar was present, a cross-sectional image crossing the midpoint of the edentulous ridge mesiodistally was chosen. If the second molar
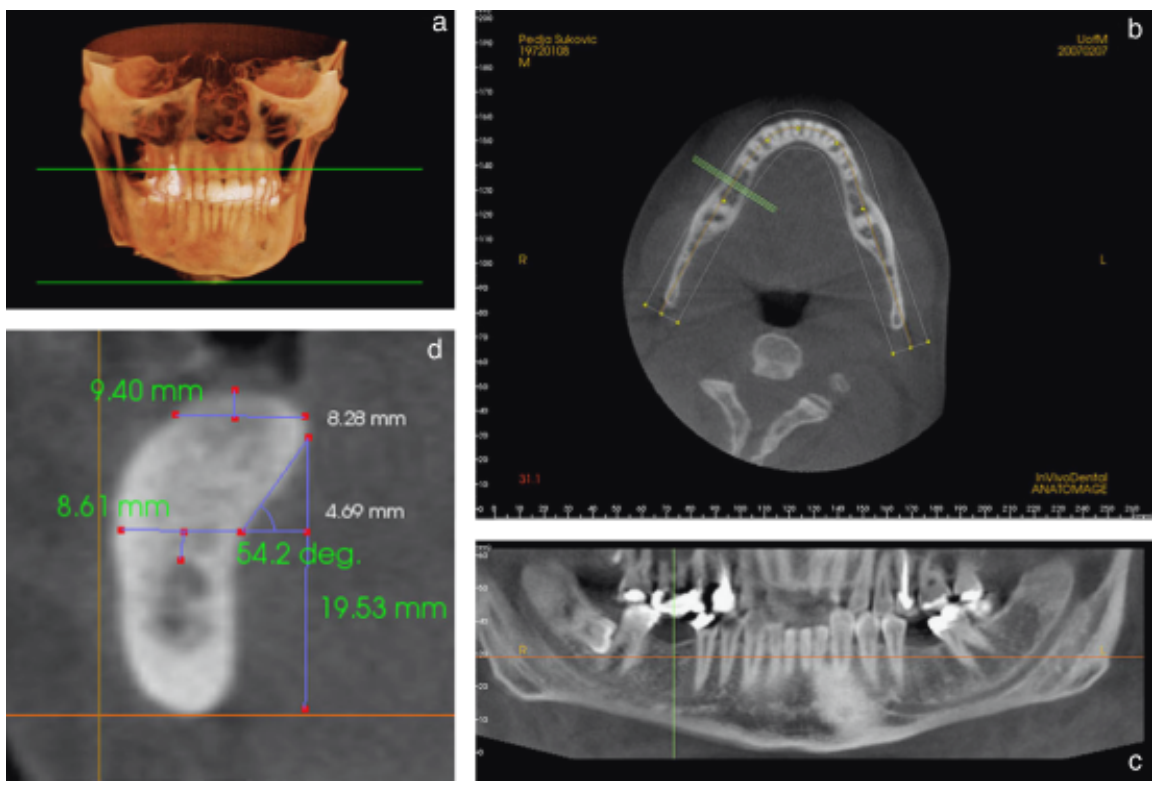

Fig. I. Schematic description of the steps of image orientation: (a) region of interest (ROI), (b) slice selection, (c) panoramic view and (d) cross-sectional view showing the relevant measurements. was absent, a cross-sectional image that was $5 \mathrm{~mm}$ distal to the cemento-enamel junction (CEJ) of the second premolar was selected instead (Fig. I).

Figure 2 summarized various measurements regarding mandible size and morphology. On the selected cross-sectional image, only the region above a horizontal line $2 \mathrm{~mm}$ coronal to the superior border of the IAN (line A) was evaluated because the implant is generally recommended to be placed with at least $\mathrm{I} .5 \mathrm{~mm}$ above the IAN (Sammartino et al. 2008). Point A was the intersection between line A and the lingual plate. For morphologic characterization of this area, the bucco-lingual width $2 \mathrm{~mm}$ apical to the alveolar crest $(\mathrm{Wc})$ and at the level of line $\mathrm{A}(\mathrm{Wb})$ was measured. The vertical distance from alveolar crest to line $\mathrm{A}(\mathrm{Vcb})$ was also measured. All variables were measured by a digital ruler provided by the same software (InvivoDent).

At the edentulous first molar region, three types of mandibular cross-sectional morphology were determined based on the following criteria (Fig. 3). A ridge with a narrow base that expands bucco-lingually to a wider crest with a prominent point (point P) (Fig. 2) on the lingual plate, giving rise to a lingual undercut, was classified as an undercut ridge type (type U). When no obvious lingual undercut was seen, the ridges were categorized into either the convergent ridge type (type C) or the parallel ridge type (type P). The type C ridge was one where the base of the ridge was wider than its crest. On the other hand, the type $P$ ridge generally had a more or less parallel ridge form. The prevalence of each ridge form was thus calculated. 


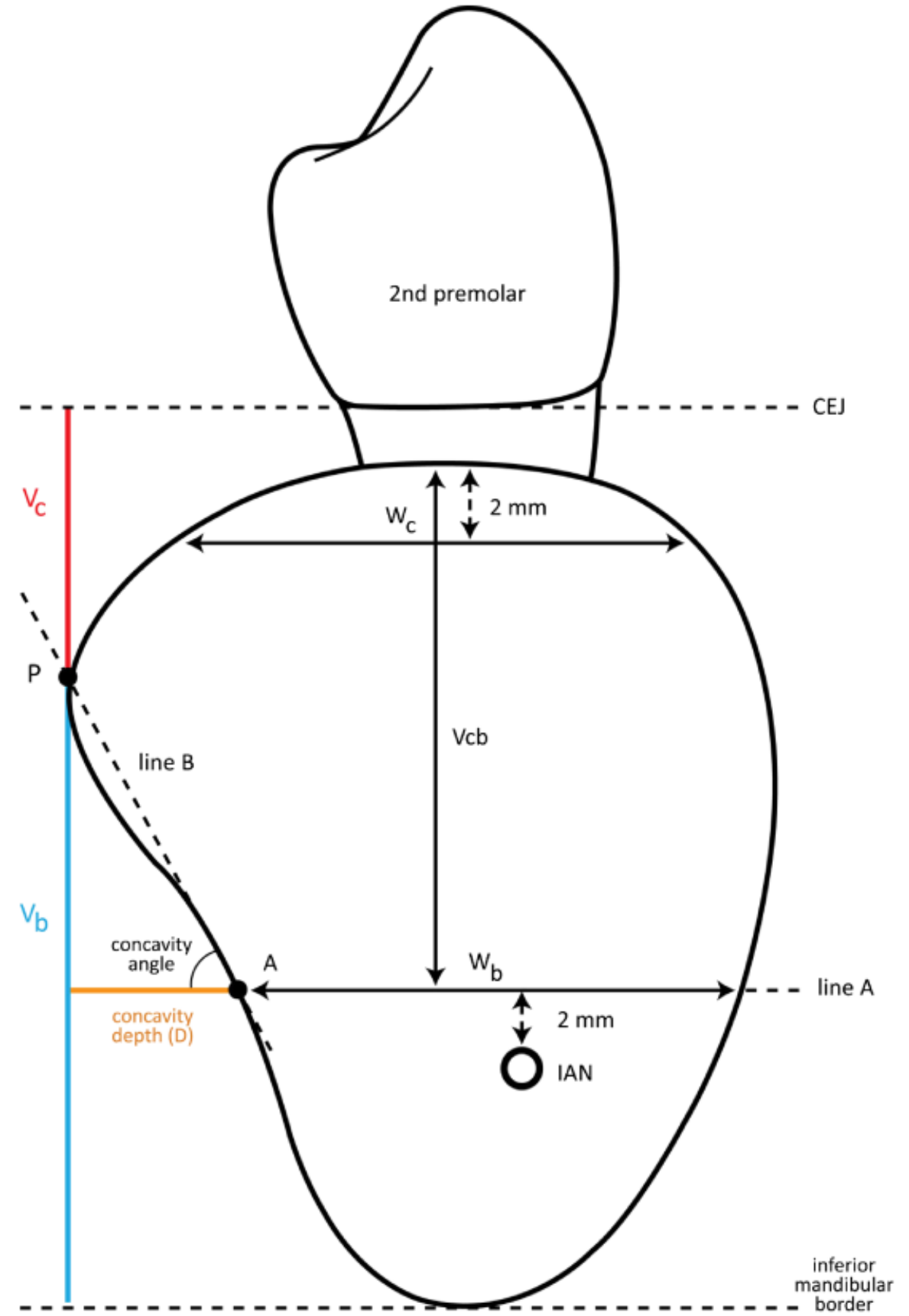

Fig. 2. The demonstration of mandibular size and lingual concavity measurements.
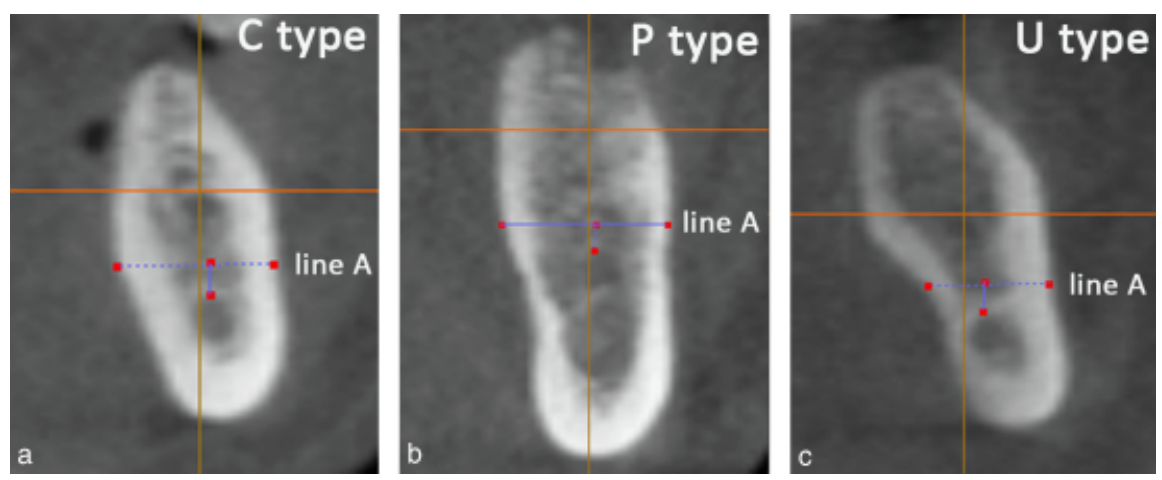

Fig. 3. Three types of cross-sectional posterior mandibular morphology: (a) C Type, (b) P Type and (c) U Type. Line A represented a reference line $2 \mathrm{~mm}$ coronal to IAN.
In type $\mathrm{U}$ ridge, the lingual concavity was measured as follows (Fig. 2). The concavity angle, in degrees, was determined by the angulation between line A and line B (the connection of point $\mathrm{A}$ and point $\mathrm{P}$ ). The linear concavity depth $(D)$ was also measured as the horizontal distance between point $\mathrm{A}$ and point $\mathrm{P}$. The greater the concavity, the smaller the angle and the greater the depth. The vertical distances of point $\mathrm{P}$ to CEJs of mandibular second premolar $(\mathrm{Vc})$ and inferior mandible border $(\mathrm{Vb})$ were also measured.

\section{Statistical analysis}

The intra-examiner agreement was determined by comparing two repeated measurements at three randomly chosen sites taken at I month apart using Pearson's correlation. As there might be a gender effect, each variable was calculated separately and compared between genders using the Student's $t$-test for independent groups. The significant level ( $P$-value) was set at 0.05 . No statistical method was applied for the categorical variable cross-sectional ridge type, and only a description of the frequencies was given. All statistical analysis was performed using a statistical package (SPSS, SPSS Inc., Chicago, IL, USA).

\section{Results}

A total of IO3 subjects were included, consisting of 35 males $(\mathrm{M})$ and 68 females $(\mathrm{F})$ with a mean age of $5 \mathrm{I}$ years (range: 23.7-70.4) and 53.2 (range: I8.4-76.9), respectively. In I8 subjects, bilateral mandibular first molar sites met the criteria, however, only one site in each subject was randomly chosen for analysis. The intraexaminer agreement ranged from 0.97 to 0.99 for the different variables. The mandibular size was summarized in Table I. The bucco-lingual width $2 \mathrm{~mm}$ apical to the alveolar crest (Wc) was $7.8 \pm 2.1 \mathrm{~mm}(\mathrm{M})$ and $6.9 \pm 2 \mathrm{~mm}(\mathrm{~F})$ and the difference between genders was significant $(P=0.03)$. The mandibular width $2 \mathrm{~mm}$ coronal to IAN $(\mathrm{Wb})$ was $10.8 \pm 2 \mathrm{~mm} \quad(\mathrm{M})$ and Io. $6 \pm 2.1 \mathrm{~mm}(\mathrm{~F})$. The vertical height from alveolar crest to $2 \mathrm{~mm}$ coronal to IAN (Vcb) was $\mathrm{I} 2.8 \pm 3 . \mathrm{I} \mathrm{mm}(\mathrm{M})$ and $\mathrm{I} 2.2 \pm 2.4 \mathrm{~mm}(\mathrm{~F})$.

In Fig. 4, a possible lingual plate perforation by an implant in mandibular first molar site was shown. The alveolar ridge on this cross-sectional view had an undercut, suggesting lingual concavity ridge type presents a risk factor of lingual perforation.

Figure 5 demonstrated the distribution of three different ridge morphology types. The type $\mathrm{U}$ ridge was the most common and $66 \%$ of this study group falling into this category. The second 
was the parallel group (type P), comprising of $20.4 \%$ and type $\mathrm{C}$ ridge was only presented in I $3.6 \%$.

The features of lingual concavity were summarized in Table I and described as follows. The concavity angle was $59.3 \pm 7.3^{\circ}(\mathrm{M})$ and $56.8 \pm \mathrm{I2}^{\circ}(\mathrm{F})$ and the linear concavity depth (D) $2.4 \pm$ I.I $\mathrm{mm}$ for both genders. The vertical distance of point $\mathrm{P}$ from the CEJ of second premolar $(\mathrm{Vp})$ and from inferior mandibular bor$\operatorname{der}(\mathrm{Vb})$ was I $2.2 \pm 2.7$ and $\mathrm{I} 5.7 \pm 2.7 \mathrm{~mm}(\mathrm{M})$ and II. $4 \pm 3 . \mathrm{I}$ and $\mathrm{I} 4.3 \pm 2.3 \mathrm{~mm} \mathrm{(F).} \mathrm{Vb}$ measurements were significantly different $(P=$ o.03) between genders.

\section{Discussion}

Quirynen et al. (2003) and Tepper et al. (200I) reported the size of the mandible using CT; however, they only focused on the interforaminal region. Recently, Watanabe et al. (2010) classified the cross-sectional mandibular morphology, including posterior region, and measured the size of the mandible. Based on the outlines of the lingual and buccal plates, three classifications, $\mathrm{A}$, $\mathrm{B}$ and $\mathrm{C}$, were described, as round on the buccal side and concave on the lingual side, concave on the buccal side and round on the lingual side, and round shape on both sides, respectively. They reported at the posterior region, type $\mathrm{C}$ (round) was the most commonly found $(59-6 \mathrm{I} \%)$, followed by type A (lingual concavity) (36-39\%). Their reported prevalence of the lingual concavity was lower than that in the present article. This might be attributed to different classification used, ethnicity (Japanese in Watanabe's study compared to mostly Caucasians and African-Americans in the present study) and the presence/absence of teeth /dentate status in
Watanabe's study). Nevertheless, both studies demonstrated a significant number of subjects with the lingual concavity.

In the same study (Watanabe et al. 2009), the width of the mandible 5-20 $\mathrm{mm}$ from the inferior border of the mandible ranged from I0.5 to
I $5.8 \mathrm{~mm}$, with no significant differences between genders. In our group, the range was wider, with 5.I-I7.I mm, although no gender differences were found.

The CBCT was introduced to the dental field to replace the cumbersome, expensive and high

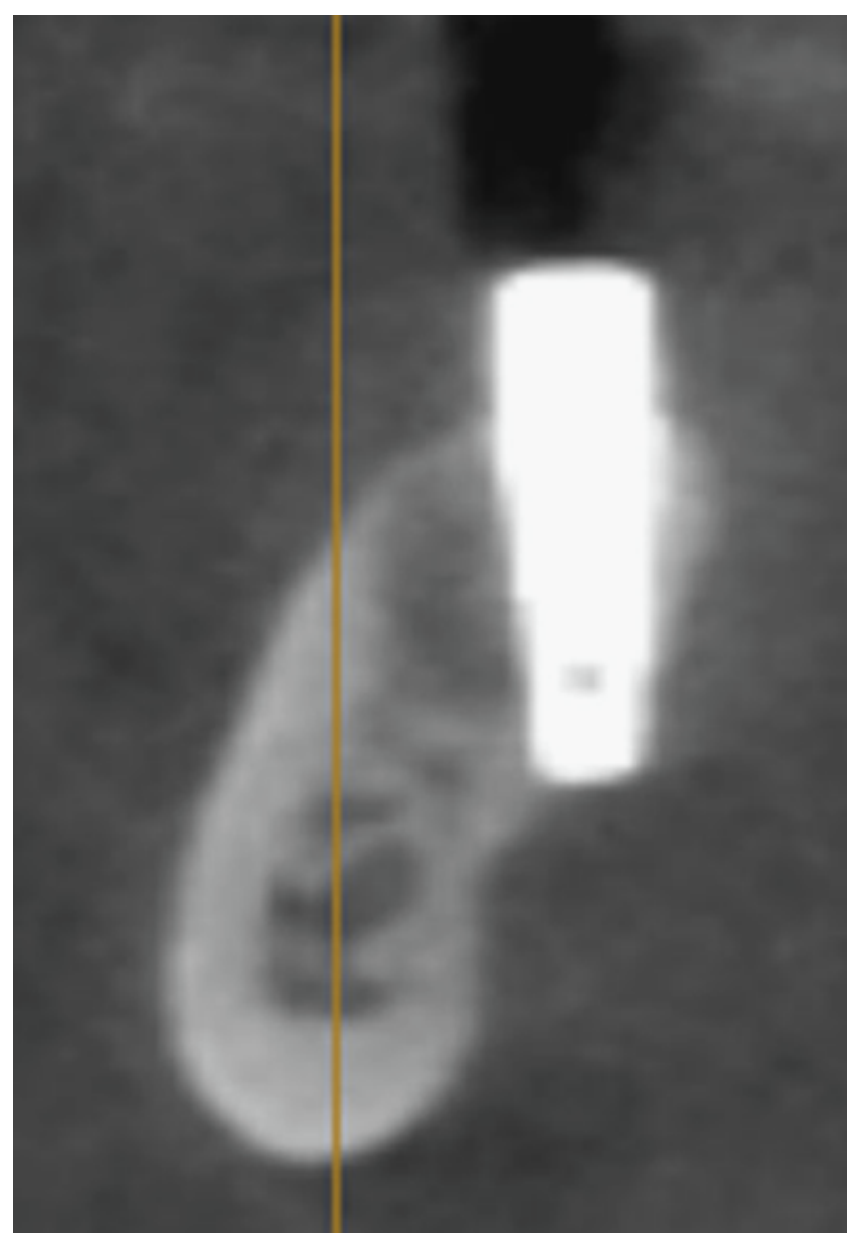

Fig. 4. The СBCT image demonstrated the possibility of lingual plate perforation by an implant.

Table 1. Measurements of mandibular dimension and lingual concavity

\begin{tabular}{|c|c|c|c|c|c|c|c|}
\hline & \multicolumn{2}{|c|}{ Bone width $(\mathrm{mm})$} & \multirow[t]{2}{*}{ Bone height $(\mathrm{Vcb})(\mathrm{mm})$} & \multirow[t]{2}{*}{ Concavity angle ( ${ }^{\circ}$ ) } & \multirow[t]{2}{*}{ Concavity depth $(D)(\mathrm{mm})$} & \multicolumn{2}{|c|}{ Vertical undercut position ( $\mathrm{mm}$ ) } \\
\hline & Wc & $\mathrm{Wb}$ & & & & Vc & $\mathrm{Vb}$ \\
\hline \multicolumn{8}{|l|}{ Male } \\
\hline$N$ (sites) & 35 & 35 & 35 & 25 & 25 & 25 & 25 \\
\hline Mean & 7.8 & 10.8 & 12.8 & 59.3 & 2.4 & 12.2 & 15.7 \\
\hline SD & 2.1 & 2 & 3.1 & 7.3 & 1.1 & 2.7 & 2.7 \\
\hline Median & 7.2 & 10.6 & 12.2 & 60.1 & 2.2 & 11.3 & 14.8 \\
\hline Range & $5.4-12.5$ & $6.9-16.2$ & $6.9-21.9$ & $36.4-76.1$ & $1.1-4.7$ & $8.4-18.2$ & $12-21.1$ \\
\hline \multicolumn{8}{|l|}{ Female } \\
\hline$N$ (sites) & 68 & 68 & 68 & 43 & 43 & 43 & 43 \\
\hline Mean & 6.9 & 10.6 & 12.2 & 56.8 & 2.4 & 11.4 & 14.3 \\
\hline SD & 2 & 2.1 & 2.4 & 12 & 1.1 & 3.1 & 2.3 \\
\hline Median & 6.5 & 10.3 & 12.2 & 56.4 & 2.1 & 11.4 & 14.3 \\
\hline Range & $3-12.8$ & $5.1-17.1$ & $7.1-17.3$ & $25-85.6$ & $0.5-5.1$ & $4.4-16.9$ & 10.6-19 \\
\hline \multicolumn{8}{|l|}{ All } \\
\hline$N$ (sites) & 103 & 103 & 103 & 68 & 68 & 68 & 68 \\
\hline Mean & 7.2 & 10.6 & 12.4 & 57.7 & 2.4 & 11.7 & 14.9 \\
\hline SD & 2 & 2 & 2.7 & 10.6 & 1.1 & 2.9 & 2.5 \\
\hline Median & 6.8 & 10.5 & 12.2 & 59.3 & 2.1 & 11.4 & 14.4 \\
\hline$P$ & 0.03 & 0.6 & 0.28 & 0.34 & 0.99 & 0.26 & 0.03 \\
\hline
\end{tabular}


Lingual concavity distribution (\%)

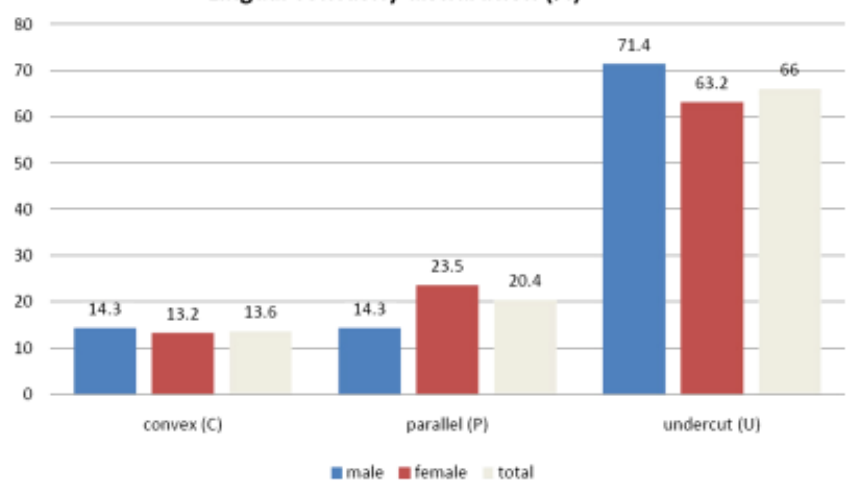

Fig. 5. Lingual concavity distribution (\%).

radiation producing medical CTs around a decade ago (Arai et al. I999). The accuracy of CBCT has been evaluated and one article (Suomalainen et al. 2008) found that the CBCT scans were more accurate than CT. In that particular article, the mean error was $4.7 \%$ (in dry mandible) and $2.3 \%$ (in sucrose solution) for CBCT images, compared with $8.8 \%$ and $6.6 \%$ for $\mathrm{CT}$ images, respectively. The ability of providing cross-sectional views of the ROI, coupled with their accuracy and high resolution, render CBCT images a good tool to assess the cross-sectional morphology of the posterior mandibular region, especially for identifying the lingual concavity.

The major potential risks of encountering a lingual plate perforation are different in the anterior and posterior mandibular regions. In the anterior mandible, branches of major arteries, for example submental and sublingual arteries, might be in close proximity to the mandible. As a result, the potential major complications in this area are massive hemorrhage (Kalpidis \& Setayesh 2004) and the possibility of subsequent airway obstruction (Givol et al. 2000), which can be fatal and thus require immediate interven-

\section{References}

Annibali, S., Ripari, M., La Monaca, G., Tonoli, F. \& Cristalli, M.P. (2009) Local accidents in dental implant surgery: prevention and treatment. The International Journal of Periodontics ↔ Restorative Dentistry 29: 325-33 I.

Arai, Y., Tammisalo, E., Iwai, K., Hashimoto, K. \& Shinoda, K. (I999) Development of a compact computed tomographic apparatus for dental use. Dentomaxillofacial Radiology 28: 245-248.

Berberi, A., Le Breton, G., Mani, J., Woimant, H. \& Nasseh, I. (I993) Lingual paresthesia following surgical placement of implants: report of a case. The International Journal of Oral $\oplus$ Maxillofacial Im plants 8: 580-582.

Brenner, D.J. \& Hall, E.J. (2007) Computed tomography-an increasing source of radiation exposure The New England Journal of Medicine 357: 2277-2284. tions. On the other hand, there are no vital structures in the submandibular space except for the submandibular glands and lymph nodes. Unless the perforation is above the mylohyoid ridge where the lingual nerve might be injured (Chan et al. 20Iо), the violation of the lingual plate in the posterior mandible does not immediately result in massive bleeding and nerve injury. However, the extruded implant may be a source of persistent inflammation or infection (if the oral mucosa is traumatized and the perforation is exposed to the oral cavity in that area). If left unattended, the infection might spread to the parapharyngeal and retropharyngeal space, leading to more severe complications, such as mediastinitis, mycotic aneurysm formation with possible subsequent rupture of the internal carotid artery, internal jugular vein thrombosis with septic pulmonary embolism or upper airway obstruction (Greenstein et al. 2008a, 2008b). Those complications may not occur immediately; however, their insidious nature warrants more attention when planning surgeries in this area.

It was demonstrated that $7 \mathrm{I} .4 \%$ of males and $63.2 \%$ of females in this study group had a mean
$2.4 \mathrm{~mm}$ lingual concavity at the level $2 \mathrm{~mm}$ coronal to the inferior alveolar nerve in the first molar region; however, the concavity can range from 0.5 to $5 . \mathrm{I} \mathrm{mm}$. The most prominent point (point $\mathrm{P}$ ) on the lingual plate, if present, was on average II.7 $\mathrm{mm}$ apical to the CEJ of the second premolar. When measured from the inferior border of the mandible, it was $14.9 \mathrm{~mm}$ coronally. These measurements may provide us with useful information regarding the ridge morphology, especially on the lingual surface, at the first molar region.

Mandibular posterior lingual concavity is a common clinical finding, which must be avoided during implant placement. Accidental perforation of the lingual plate may create a perplexing situation for both the surgeon and the patient. If left unattended, inflammation and infection may ensue. The results regarding the prevalence, position and extent of the lingual concavity in this article provide additional information for safe implant placement in mandibular first molar area. Future research should focus on the identification of factors, which may predict the degree of lingual concavity and risks of posterior lingual plate perforation.

Acknowledgements: We would like to thank Dr Erika, Benavides, Clinical assistant professor, Department of Periodontics \& Oral Medicine, School of Dentistry, University of Michigan for her expertise and Chia-Ning Wang, PhD student, Department of Biostatistics, School of Public Health, University of Michigan for statistical analysis consultation. This paper was partially supported by the University of Michigan Periodontal Graduate Student Research Fund.
Chan, H.L., Leong, D.J., Fu, J.H., Yeh, C.Y., Tatarakis, N. \& Wang, H.L. (20IO) The significance of the lingual nerve during periodontal/implant surgery. Journal of Periodontology 81: 372-377.

Chen, L.C., Lundgren, T., Hallstrom, H. \& Cherel, F. (2008) Comparison of different methods of assessing alveolar ridge dimensions prior to dental implant placement. Journal of Periodontology 79: 40I405.

Chiapasco, M., Abati, S., Romeo, E. \& Vogel, G. (I999) Clinical outcome of autogenous bone blocks or guided bone regeneration with e-PTFE membranes for the reconstruction of narrow edentulous ridges. Clinical Oral Implants Research го: 278-288.

Givol, N., Chaushu, G., Halamish-Shani, T. \& Taicher, S. (2000) Emergency tracheostomy following lifethreatening hemorrhage in the floor of the mouth during immediate implant placement in the mandibular canine region. Journal of Periodontology $7 \mathbf{I}$ : I893-I895.

Greenstein, G., Cavallaro, J., Romanos, G. \& Tarnow, D. (2008a) Clinical recommendations for avoiding and managing surgical complications associated with implant dentistry: a review. Journal of Periodontology 79: $1317-1329$.

Greenstein, G., Cavallaro, J. \& Tarnow, D. $(2008 \mathrm{~b})$ Practical application of anatomy for the dental implant surgeon. Journal of Periodontology 79: I833I 846 .

Kalpidis, C.D. \& Setayesh, R.M. (2004) Hemorrhaging associated with endosseous implant placement in the anterior mandible: a review of the literature. Journal of Periodontology 75: 63 I-645.

Lekholm, U. \& Zarb, G. (I985) Patient selection and preparation. In: Branemark, P.I., Zarb, G.A. \& Al- 
brektsson, T., eds. Tissue Integrated Prosthesis: Osseointegration in Clinical Dentistry. Chicago: Quintessence Publications Co. Inc., I99 pp.

Quirynen, M., Mraiwa, N., van Steenberghe, D. \& Jacobs, R. (2003) Morphology and dimensions of the mandibular jaw bone in the interforaminal region in patients requiring implants in the distal areas. Clinical Oral Implants Research I4: $280-285$.

Sammartino, G., Marenzi, G., Citarella, R., Ciccarelli, R. \& Wang, H.L. (2008) Analysis of the occlusal stress transmitted to the inferior alveolar nerve by an osseointegrated threaded fixture. Iournal of Periodontology 79: I735-I744.

Suomalainen, A., Vehmas, T., Kortesniemi, M., Robinson, S. \& Peltola, J. (2008) Accuracy of linear measurements using dental cone beam and conventional multislice computed tomography. Dentomaxillofacial Radiology 37: IO-I7.

Tepper, G., Hofschneider, U.B., Gahleitner, A. \& Ulm, C. (200I) Computed tomographic diagnosis and localization of bone canals in the mandibular interforaminal region for prevention of bleeding complications during implant surgery. The Interna- tional Journal of Oral ↔) Maxillofacial Implants I6: 68-72.

Tyndall, D.A. \& Brooks, S.L. (200o) Selection criteria for dental implant site imaging: a position paper of the American Academy of Oral and Maxillofacial Radiology. Oral Surgery, Oral Medicine, Oral Pathology, Oral Radiology et Endodontics 89: 630-637.

Watanabe, H., Mohammad Abdul, M., Kurabayashi, T. \& Aoki, H. (20ıо) Mandible size and morphology determined with CT on a premise of dental implant operation. Surgical Radiologic Anatomy 32: 343349 . 